\title{
VI. Nach Straßers Rücktritt
}

\section{1. Änderung der Parteiorganisation}

Am 9. Dezember 1932, ${ }^{1}$ also bereits am Tage nach Straßers Ausscheiden aus seinen Parteiämtern, übernahm aber Hitler selbst die Leitung der Reichsorganisationsabteilung. $\mathrm{Zu}$ seinem Stellvertreter und damit zum eigentlichen Leiter der ROL machte er den bisherigen Reichsinspekteur Ley mit dem neuen Titel eines Stabsleiters der PO. Eiligst begann Hitler Straßers Machtapparat zu zerschlagen, wobei er allerdings vorsichtig bemüht blieb, nicht direkt Stellung gegen Straßer zu beziehen; er erwähnte nicht einmal seinen Namen. Die von Hitler vorgenommene Umstrukturierung betraf einmal die vertikale Gliederung der Partei, zum anderen die Ressorts der ROL. In vier verschiedenen Verfügungen vom 14. und 15. Dezember 1932, die im VB veröffentlicht wurden, gab Hitler die Neugliederung der Partei bekannt. $^{2}$ Intern erläuterte er seine Anordnungen noch in einer zweiteiligen Denkschrift vom 15. und 20. Dezember 1932. ${ }^{3}$

Zunächst wurden die Reichsinspektionen abgeschafft, was personell insofern unproblematisch war, als Reichsinspekteur II Ley durch die Neuorganisation zum Stabsleiter, d. h. praktisch zum Nachfolger Straßers aufrückte. Die Landesinspekteure sollten alle in ihre früheren Gauleitungen zurückkehren und erhielten die Stellung eines Kommissars für das Gebiet ihrer ehemaligen Landesinspektion. Soweit bereits unter Straßer Nachfolger für die zu Landesinspekteuren beförderten Gauleiter ernannt worden waren, kam es sicherlich zu Schwierigkeiten, mußten doch die neuernannten Gauleiter ihre Posten für die nun zurückkehrenden Landesinspekteure wieder räumen. Jedoch waren sie in ihren Stellungen noch zu neu und ungefestigt, als daß sie größeren Widerstand hätten leisten können. Von den hier wichtigen vier Fällen ist lediglich einer in den Akten zu verfolgen, und zwar der Fall Sprenger Linder. Nachdem Sprenger im Juni 1932 zum Landesinspekteur ernannt worden war, wurde sein stellvertretender Gauleiter Karl Linder nach einigen Querelen am 17. August 1932 von Straßer zum Gauleiter Hessen-Nassau bestellt. Jetzt mußte er den Platz wieder räumen. Ohne daß die Gründe ersichtlich sind, trat er aber erst am 31. Dezember 1932 zurück und übernahm wieder die Stellung des stellvertretenden Gauleiters. Offenbar zur endgültigen Bereinigung wurde er am 12. März 1933 zum Bürgermeister der Stadt Frankfurt a. Main ernannt. ${ }^{4}$ Ein Sonderfall war der Landes-

1 VB, 11./12. 12. 1932.

2 VB, 16. u. 17. 12. 1932.

3 Denkschrift über die inneren Gründe für die Verfügungen zur Herstellung einer erhöhten Schlagkraft der Bewegung: Teil I v. 15. 12. 1932 (BA, NS 22/110), Teil II v. 20. 12. 1932 (BA, NS 22/356).

${ }^{4}$ Personalakte Linder, BDC. 
inspekteur West, Heinz Haake: Da er vorher keinen eigenen Gau gehabt hatte, konnte er auch jetzt keine Gauleiterposition zurückbekommen. Er wurde zum Kommissar - West ernannt und gleichzeitig dem Stabsleiter Ley zur besonderen Verfügung gestellt.

Zu den Aufgaben eines Kommissars ordnete Hitler an: „Die kommissarische Tätigkeit findet nur statt auf Anordnung der Reichsleitung bzw. in meinem Auftrage oder im Auftrage des Stabsleiters der PO.“ In der internen Denkschrift ging Hitler ausführlich auf die Stellungen der Gauleiter und der Kommissare ein. Den Gauleitern wurde eine Immediatstellung bei Hitler zugesichert. Klar und deutlich erklärte er, daß die Gauleiter nicht, wie alle anderen Amtswalter der Partei, der ROL, sondern allein dem Führer unterständen. ,Der Gauleiter ist in seinem Bereich der Repräsentant der Bewegung. Er wird dieser Aufgabe um so mehr nachkommen können, je mehr in der Organisation selbst die Bedeutung seiner Stellung zum Ausdruck kommt.“ Die Bedeutung der Stellung sollte nach den Worten Hitlers gerade darin ihren Ausdruck finden, daß keine Zwischeninstanz, wie etwa die früheren Landesinspektionen, sie mehr von ihm, dem Führer, trennte. So sollten auch die Kommissare, wie die bisherigen Inspekteure nun genannt wurden, möglichst ,,selbst als Gauleiter in der Front der Bewegung verankert sein". Der Titel sollte die Kommissare aus den übrigen Gauleitern nur insoweit herausheben, als er sie als besonders erfahrene und Hitler besonders ergebene Mitarbeiter auszeichnete. Eine Funktion war normalerweise nicht damit verbunden. Nur in besonderen Ausnahmefällen sollten die Kommissare als „Bevollmächtigte der Reichsleitung“ für kurze Zeit auf Aufforderung des Führers in die Nachbargaue eingreifen können, um dort etwaige kritische Situationen, etwa beim völligen Versagen eines Gauleiters, auffangen zu können. Die Funktionslosigkeit der Kommissare zeigte sich auch darin, daß ihnen mit Ende des Monats Dezember 1932 der bis dahin für die Landesinspekteure gewährte Etat durch Reichsschatzmeister Schwarz gestrichen wurde. Nur Kommissar-West, Haake, erhielt weiter den bisherigen Etat, da er keine Gauleiterposition innehatte; von den übrigen heißt es lakonisch, daß sie ,ihre Auslagen als Kommissare aus der Gaukasse decken" müssen. ${ }^{5}$

Dann wandte sich Hitler dem großen ,zentralen Organisationsapparat“ der ROL zu. ${ }^{6}$ Wiederum ohne Straßer persönlich anzugreifen, kritisierte er den Apparat der ROL grundsätzlich. So polemisierte er gegen die Größe der ROL: „Es ist auch ein Irrtum anzunehmen, die Organisation wäre umso besser, je umfangreicher und gegliederter ihr Apparat in Erscheinung tritt." Auch einen Seitenhieb auf die Fachleute, die Straßer um sich versammelt hatte und die ihm bei der Ausarbeitung von programmatischen Aussagen, Reden oder Gesetzesvorlagen halfen, konnte sich Hitler nicht verkneifen: „Wissenschaftliche Forschungsinstitute auf mehr oder weniger abseits liegenden Gebieten gehören nicht in den politischen Organisationsapparat.“ An anderer Stelle sagte er: „Ganz besonders aber ist die Organisation der Partei

5 Brief Ley an alle Kommissare v. 21. 12. 1932, BA, NS 22/7.

6 Vgl. Anm. 3. 
kein Betätigungsfeld für wissenschaftliche Experimente. Ob und welche technische revolutionäre Umwälzung die Partei einst durchführen wird, kann nicht in den Schreibstuben einer Organisationsabteilung entschieden werden. Denn dies hieße die Bewegung von ihrer weltanschaulichen Mission immer mehr entfernen und damit an die Stelle ihrer ewig richtigen weltanschaulichen Grundsätze ewig schwankende und unsichere wissenschaftliche Theorien setzen." Im einzelnen wurden folgende Veränderungen in der ROL verfügt:

1. Die Abteilung für Volksbildung, bisher eine Unterabteilung der Hauptabteilung III der ROL, wurde in die Reichspropagandaabteilung eingegliedert und Goebbels unterstellt, der damit eine wesentliche Abrundung seiner Kompetenzen auch im Hinblick auf sein späteres Ministerium erhielt.

2. Die innenpolitische Abteilung, bisher ebenfalls in der Hauptabteilung III, wurde der Rechtsabteilung zugeschlagen.

3. Der agrarpolitische Apparat, bisher Hauptabteilung V der ROL, wurde mit allen angeschlossenen Abteilungen als selbständige Reichsleitung dem Führer direkt unterstellt.

4. Die Wirtschaftspolitische Abteilung, bisher Hauptabteilung IV der ROL, wurde ebenso wie der Reichswirtschaftsrat aufgelöst.

Um einige Kompetenzen der alten ROL aufzufangen, rief Hitler eine politische Zentralkommission ins Leben, die unmittelbar dem Stellvertreter des Führers, Rudolf Hess, unterstand. Sie unterteilte sich wiederum in:

1. Kommission für Beratung und Überwachung der nationalsozialistischen parlamentarischen Arbeit in den Länderparlamenten und Kommunen; Vorsitzender Dr. Buttmann

2. Kommission zur Überwachung der nationalsozialistischen Presse; Vorsitzender Dr. Dietrich

3. Kommission für Wirtschaftsfragen;

Vorsitzender der Abteilung Wirtschaftspolitik, Funk

Vorsitzender der Abteilung Arbeitsbeschaffung, Feder

$\mathrm{Zu}$ den der ROL unter Robert Ley verbliebenen Aufgaben bemerkte Hitler in seiner Denkschrift: „Es muß daher unser Ziel sein, das politische Organisationsamt (gemeint ist die ROL, der Verf.) auf seine wenigen sinngemäßen Aufgaben zu beschränken: 1. Personal-Politik (Personalamt, Führerkartothek), 2. Parteigliederung, 3. Parteischulung (nicht zu verwechseln mit den Aufgaben der Propaganda).“ Es blieb Ley also aus Straßers Machtappparat neben der NSBO bzw. später der $\mathrm{DAF}^{7}$ im wesentlichen nur die Verwaltung der Partei und die Schulung der Funktionäre, wobei die Gauleiter grundsätzlich, da Hitler unmittelbar unterstellt, ausgenommen waren. Hitler hofierte die Gauleiter regelrecht, wenn er feststellte: „Es ist mein Wunsch und Wille, daß ihre Stellung in der Bewegung eine möglichst souveräne ist. Mögen sie sich aber auch selbst in der Partei als ebenso souveräne Repräsentanten der Partei fühlen und führen."

7 Hüttenberger, a. a. O., S. 131 ff. 
Am Ende der Denkschrift hielt Hitler den Gauleitern und anderen führenden Männern der Parteispitze noch ein Ziel vor Augen, das sie sozusagen bei guter Führung erreichen könnten: den Parteisenat. Der kleine Senat sollte unter Hitlers Führung 24 Mitglieder haben, seine Aufgabe darin bestehen, ,in Voll- und Teilsitzungen die gemeinsam berührenden wichtigen Parteifragen durchzusprechen und zu behandeln". Daneben war an einen großen Parteisenat mit 64 Mitgliedern gedacht: „Durch ihn soll eine Anzahl der ältesten, treuesten und fähigsten Köpfe der Bewegung in unmittelbare Verbindung mit der obersten Parteileitung gebracht werden. $\mathrm{Zu}$ diesem großen Senat der Bewegung zu gehören, soll in Zukunft als größte Ehre der Bewegung empfunden und angesehen werden." Außerdem sollte dem großen Senat nach Hitlers Willen eine durchaus bedeutende Aufgabe zufallen. Er hielt die Bildung des großen Senats ,,schon deshalb für wichtig, damit im Falle meines Todes die Frage des neuen Führers der Partei nicht in einem wilden Kampf unter den Parteigenossen entschieden wird, sondern von dem Senate aus seine Entscheidung findet".

\section{Die Erschütterung in der NSDAP}

Auf Grund der Neuorganisation der NSDAP notierte Goebbels bereits am 12. Dezember 1932 in seinem Tagebuch: „Straßer hat auf der ganzen Linie verloren. " ${ }^{8} \mathrm{Da} \beta$ dies jedoch etwas voreilig war, lie $\beta$ er in weiteren Eintragungen erkennen. Vom 10. Dezember 1932 bis Anfang Januar 1933 verging kaum ein Tag, an dem er nicht von Amtswaltertagungen oder anderen parteiinternen Versammlungen zu berichten hatte, auf denen Hitler und er im gesamten Reichsgebiet die Parteifunktionäre über die Lage aufklären und dadurch von Straßer ablenken mußten. ${ }^{9}$ In der Tat verlief die Krise völlig anders als vergleichbare frühere Erschütterungen der Partei. Sowohl in der Otto-Straßer-Krise von 1930 als auch 1931 beim StennesAbfall hatten jeweils die Führungspersonen, also Otto Straßer und der SA-Führer Stennes, aktiv zum Abfall von München aufgerufen und versucht, alle ihnen bekannten oder verbundenen NS-Mitglieder, insbesondere auch Amtsträger der Partei, zu sich herüberzuziehen. Anders Gregor Straßer, der bei seinem Abschied sowohl in seiner Rede vor den Landesinspekteuren ${ }^{10}$ wie auch in seinem Rücktrittsbrief ${ }^{11}$ eindeutig erklärte, daß sein Rücktritt eine persönliche Entscheidung sei und er alle anderen auffordere, auf ihren Posten in der Partei zu bleiben. Anschließend war er für mehrere Wochen in Urlaub verschwunden und hatte sich damit bewußt jeder Möglichkeit, Kristallisationskern einer Hitler-Fronde zu werden, entzogen. Während aber 1930 und 1931 die Bemühungen der Parteirebellen an der Integrations-

${ }^{8}$ Goebbels, a. a. O., S. 224.

9 Ebenda, S. $223 \mathrm{ff}$.

10 Lohse-Bericht.

11 Schulz-Memorandum, siehe Anhang. 
kraft des Hitlermythos scheiterten, ${ }^{12}$ war der Glaube an die politische Unfehlbarkeit des Führers durch die vielfältigen Rückschläge und Mißerfolge des Jahres 1932 so stark erschüttert, daß die Krise nicht so ohne weiteres überwunden werden konnte. Durch den plötzlichen Rücktritt überrascht und durch die politische Abstinenz des unauffindbaren Straßer ihres Mittelpunktes beraubt, verharrten seine zahlreichen Anhänger zunächst in konsterniertem Abwarten. Viele blieben in ihren Positionen, um bei der erwarteten Rückkehr Straßers bereit zu sein. ${ }^{13}$ Bald aber begannen Straßer-Anhänger und allgemein unzufriedene NS-Mitglieder auf regionaler Basis in der Rolle einer Parteiopposition aufzutreten und eine Änderung der Hitlerschen Politik zu fordern, wobei sie sich mehr oder weniger offen auf Straßer und dessen Konzeption beriefen. Die Ansätze einer Parteirevolte zeigten sich an verschiedenen Stellen fast gleichzeitig, ohne daß die einzelnen Aktionen koordiniert waren und ohne daß die Zentralfigur der Bewegung aktiven Anteil an ihr nahm. Die kurz danach erfolgte Machtergreifung Hitlers am 30. Januar 1933 entzog dann allerdings dieser Bewegung den Boden, die ja auch an vielen Orten noch nicht über das Vorbereitungsstadium hinausgekommen war. Oft wurden die Spuren solcher Aktivitäten auch von den Initiatoren im Laufe der Festigung der NS-Herrschaft beseitigt, so daß sie heute nicht mehr oder nur noch schemenhaft zu erkennen sind. Jedoch sind die heute noch nachweisbaren Fälle interessant und beispielhaft genug, um eine beginnende Auflösung der Hitlerschen NSDAP als Folge des Straßer-Rücktritts und bei längerer Erfolglosigkeit Hitlers immerhin möglich erscheinen zu lassen.

\section{a) Das „Freikorps Franken“}

Am bekanntesten, wenn auch noch nicht erschöpfend untersucht, ist die Revolte des SA-Gruppenführers und NS-Reichstagsabgeordneten Wilhelm Stegmann, die im Januar 1933 zur Gründung des „Freikorps Franken“ führte. Der Diplomlandwirt und Pächter der Domäne „Schillingsforst" in Franken zählte zur alten Garde der Nationalsozialisten. Am 14. Dezember 1925 war er mit der Parteinummer 24713 in die NSDAP eingetreten. Seitdem hatte er zu den eifrigsten Propagandisten und Organisatoren der NSDAP und der SA in den ländlichen Gebieten Frankens gehört. Die enge Zusammenarbeit mit der Reichspropagandaleitung in den Jahren 1928 bis 1930 hatte ihm die Duzfreundschaft Himmlers eingebracht. 1930 war er als Abgeordneter der NSDAP in den Reichstag eingezogen. In der SA stieg er zum Führer der SA-Gruppe Franken auf. Im Laufe des Jahres 1932 häuften sich die Schwierigkeiten der fränkischen SA-Führung mit der Nürnberger Gauleitung unter Julius Streicher, dem von der SA „Bonzentum“ und „Korruption" vorgeworfen wurde. Im Spätherbst wurde dieser Streit so heftig, daß die Parteileitung am 9. Dezember 1932 die SA-Untergruppe „Mittelfranken“, d. h. den Nürnberger Raum, aus der SA-

12 Horn, a. a. O., S. $299 \mathrm{ff}$.

13 Auskunft Dr. Hagert v. 18. 5. 1971; Personalakte Reupke, BDC. 
Gruppe Franken und damit aus Stegmanns Befehlsgewalt herauslöste, um dadurch die Reibungsflächen mit Streicher zu vermindern. ${ }^{14}$

Der Konflikt war jedoch, zumal vor dem Hintergrund der jetzt offen ausbrechenden Straßerkrise, bereits zu weit gediehen, als daß er mit rein organisatorischen Maßnahmen hätte beigelegt werden können. Die SA-Funktionäre, die sich verbal stets mit Hitler solidarisierten, griffen Streicher und seine „Bonzen“ immer heftiger an und forderten demonstrativ deren Entlassung. Als daraufhin die Parteileitung Stegmann seines Postens enthob und weitere Unterführer maßregelte oder gar ausschlo $\beta,{ }^{15}$ wurde dies von der fränkischen SA mit der Bildung des ,Freikorps Franken“ beantwortet, an dessen Spitze sich Stegmann, freilich erst nach einigem Zögern, gestellt hatte. Dies führte am 20. Januar 1933 zu seinem Parteiausschluß. Schienen zunächst örtliche und auch persönliche Querelen Anlaß des Konflikts gewesen zu sein, so äußerte sich Stegmann nach seinem Ausschluß deutlicher über die wahren Gründe. In einer öffentlichen Versammlung sagte er am 24. Januar 1933, daß nach seiner Auffassung die Partei 1932 ihren geschichtlichen Augenblick verpaßt habe und offenbar deshalb in ein gefährliches Stadium hineingeschlittert sei. Mit der Parole „Der Kampf geht weiter!“ sei heute nichts mehr getan. Notwendig sei vielmehr ein neuer revolutionärer Aufbruch der Partei mit sozialem Einschlag. ${ }^{16}$ Ohne den Namen zu nennen, hatte er damit genau Straßers Position in dessen Zerwürfnis mit Hitler bezogen.

Im Gegensatz zur Stennes-Affäre, die organisatorisch wesentlich daran gescheitert war, daß die SA-Unterführer loyal zu Hitler gestanden hatten, beteiligten sich diesmal nahezu alle fränkischen Unterführer an der Revolte. ${ }^{17}$ Das Ausmaß dieser Oppositionsbewegung der fränkischen SA ist ungefähr an den Ausschlüssen zu erkennen, die im „Verordnungsblatt der Obersten SA-Führung“"vom 1. Februar 1933 verkündet wurden. ${ }^{18}$ Während unter "Ausschließung“ in anderen SA-Gruppen durchweg nur einfache SA-Männer oder, ganz selten, niedrige Chargen genannt werden, ergibt sich für Franken folgendes Bild: Neben dem Gruppenführer Stegmann wurden sieben Standartenführer, ${ }^{19}$ vierzehn Sturmbannführer und vierzehn Sturmführer ausgeschlossen. Im Januar 1932 zählte Franken 6711 SA-Mitglieder. ${ }^{20}$ Angesichts der Gesamtentwicklung kann daher für die Jahreswende 1932/33 ein Mitgliederstand von 7-8000 Männern angenommen werden. Das „Freikorps Franken“ zählte gegen Ende Januar 1933 etwa 6-7000 Mitglieder, und es trafen noch „täglich Neuanmeldungen" ein. Also war nahezu die gesamte fränkische SA zu

14 Horn, a. a. O., S. 411.

$15 \mathrm{VB}, 21 . / 22.1 .1933$.

16 „Münchner Zeitung“", 25. 1. 1933.

17 Brief Reupke an Elbrechter vom 24. 1. 1933 (Elbrechter-Nachlaß): „Wesentlich ist, daß so gut wie sämtliche Unterchargen von Stegmann zu ihm übergetreten sind.“

18 „Verordnungsblatt der Obersten SA-Führung“, 1.2. 1933, BA, NS 26/308.

19 Da eine Standarte etwa 1000-3000 Mann Stärke haben sollte (siehe Tyrell, a.a. O., S. 354), die SA-Franken damals aber 7000-8000 Mitglieder hatte, müssen dies sämtliche Standartenführer gewesen sein.

20 „Der Oberste SA-Führer“, München, den 27. 2. 1932, BA, NS 26/307. 
Stegmann übergelaufen. ${ }^{21}$ Auch aus der SS kam Zulauf, wiewohl offenbar im wesentlichen nur niedere Chargen. 22

Stegmann versuchte auch sofort, Verbindungen herzustellen, die das politische Gewicht seiner Gruppierung stärken sollten; ferner bemühte er sich finanzielle Unterstützung zu erschließen, und zwar über den Rechtsanwalt Dr. Reupke, Syndikus des Reichsverbandes der deutschen Industrie und enger persönlicher Freund und politischer Berater Gregor Straßers. Reupke suchte den Ruhrbergbau finanziell für die Stegmann-Aktion zu gewinnen, ${ }^{23}$ auch berichtete er über Elbrechter laufend Schleicher über die Entwicklung des Freikorps, um dadurch den Reichskanzler zur finanziellen Hilfe für Stegmann zu veranlassen. ${ }^{24}$ Für den 29. Januar 1933 vermittelte Reupke schließlich ein geheimes Treffen zwischen Stegmann, Gregor Straßer und Schulz in München. ${ }^{25}$

Organisatorisch begann sich das „Freikorps Franken“ schnell zu festigen. In bewährter SA-Manier wurden ,ständige Sprechabende“ in bestimmten Lokalen zu festen Zeiten eingerichtet: „Eintritt frei. Juden haben keinen Zutritt! Ruhestörer fliegen raus!" Daneben wurden Verkehrslokale angegeben und ein Hilfswerk eingerichtet, das an Erwerbslose Essen ausgeben und für Obdachlose Unterkünfte bereitstellen sollte. Auch Appelle der einzelnen Kompanien wurden festgesetzt. Zur Erweiterung der Basis entstand bald noch eine ,Frauen-Arbeits-Gemeinschaft des Freikorps““ ${ }^{26}$ Im Januar 1933 erschien dann die erste Nummer einer eigenen Zeitschrift: „Das Freikorps, Herausgeber Wilhelm Stegmann, Kampfblatt für Sauberkeit und Reinheit der Nationalsozialistischen Idee." Die Schlagzeilen der ersten Ausgabe lauteten: „Kameraden - Nationalsozialisten. Nun erst recht! Licht im Bonzen-Nebel.“ Noch im Januar 1933 versuchte Stegmann aber auch, die territoriale Basis seiner Bewegung zu verbreiten, und zwar zunächst offensichtlich mit Erfolg. So bereitete er Berichte über die Ereignisse, die zur Bildung des „Freikorps“ geführt hatten, vor, um sie an alle SA- und SS-Gruppenführer zu schicken; sie sollten in seinem Sinne unterrichtet werden. ${ }^{27}$ Dann trat er mit einzelnen SA-Verbänden in direkten Kontakt, um sie zum Anschluß zu bewegen. Zuerst verhandelte er mit der Standarte Regensburg, die ihm Ende Januar auch zusicherte, sich ihm anzuschließen. ${ }^{28}$ Am 27. Januar 1933 lag in Berlin die Nachricht von Stegmann vor, daß sich ihm auch eine Standarte in der Pfalz und Teile der Hitlerschen Leibstandarte angeschlossen hätten. ${ }^{29}$ Auch in der Berliner SA erregte die Stegmann-Parole ,für Sauberkeit und Ordnung“ Aufsehen. Offensichtlich waren hier Ende Januar einige Gruppen, in

21 Brief Reupke, siehe Anm. 17.

22 Personalakte Reupke, BDC; Personalakte Stegmann, BDC.

23 Brief Reupke an Heinrichsbauer vom 26. 1. 1933, Personalakte Reupke, BDC.

24 Brief Reupke, vgl. Anm. 17.

25 Brief Reupke an Schulz vom 27. 1. 1933, Personalakte Reupke, BDC.

26 Zeitschrift „Das Freikorps“, 1. 2. 1933.

27 Brief Reupke, vgl. Anm. 17.

28 Ebenda.

29 Brief Reupke, vgl. Anm. 25. 
denen neben der allgemeinen Unruhe auch noch Erinnerungen an Stennes schwelten, bereit, sich in den nächsten Tagen oder Wochen dem Freikorps anzuschließen. ${ }^{30}$ Gleichzeitig tauchten Gerüchte auf, daß in Westdeutschland die Gründung eines „Freikorps Ruhr" unmittelbar bevorstehe. ${ }^{31}$

Das „Freikorps Franken“ hielt sich noch einige Monate nach der Machtergreifung. Dann löste es sich langsam wieder auf. Viele Mitglieder suchten und fanden einzeln den Weg zurück in die NSDAP, während sich der Rest im Laufe der Festigung des Hitler-Regimes verlor.

\section{b) Die Notgemeinschaften}

Aber nicht nur in der SA gärte es, auch in der Politischen Organisation machten sich Auflösungserscheinungen bemerkbar. Wie Gauleiter Wagner (Baden) in einem vertraulichen Bericht an die Reichs-Uschla schrieb, ${ }^{32}$ bildeten sich im Winter 32/33 im ganzen Reich Oppositionsgruppen innerhalb der Partei, die durchweg unter dem Namen „Notgemeinschaften“ arbeiteten. Das Material der Notgemeinschaft in Lahr, die in den Gauen Baden und Hessen-Nassau-Süd arbeitete, ist zum Teil erhalten geblieben. Zentrale Figuren dieser Notgemeinschaft waren der Reichstagsabgeordnete Karl Lenz, gleichzeitig Gauleiter von Hessen-Darmstadt, der Reichstagsabgeordnete Fritz Kern und Dr. Werner Best, ${ }^{33}$ der Autor der sogenannten Boxheimer-Dokumente. ${ }^{34}$ Eigentlicher Organisator der Gruppe war aber der bereits am 20. Oktober 1932 ausgeschlossene $e^{35}$ ehemalige Kreisleiter von Mannheim, der Ingenieur Felix Wankel. Schon im November 1932 begann Wankel zusammen mit einigen anderen oppositionellen NS-Mitgliedern eine eigene Zeitung herauszugeben, die unter dem Namen „Alemannische Grenzlandnachrichten (Algrena)“ zweimal wöchentlich erschien und abweichende Ideen unter dem nationalsozialistisch orientierten Teil der Bevölkerung verbreitete. ${ }^{36}$ Ende November stieß Gauleiter Lenz zu dieser Gruppe. Nachdem Straßer am 8. Dezember zurückgetreten war, legte Lenz etwa Mitte Dezember 1932 seinen Gauleiterposten nieder und zog nach Heidelberg. ${ }^{37}$ Dort begann er Artikel für die „Algrena“ zu schreiben, in denen er sich mit dem Rücktritt Straßers befaßte. Außerdem reiste er in den Gauen Baden und Hessen-Darmstadt herum und versuchte, gleichgesinnte NS-Mitglieder zu finden. Zur

30 Ebenda.

31 Brief Reupke an Heinrichsbauer vom 26. 1. 1933 und Antwort Heinrichsbauer an Reupke vom 27. 1. 1933, Personalakte Reupke, BDC.

32 Personalakte Lenz, BDC. Der Bericht des Gauleiters Wagner ist undatiert, gehört aber in die Uschla-Akten des Monats August 1933, vgl. IfZ, MA 329, Blatt 652261.

33 Personalakte Lenz, BDC: Zeugenvernehmungen.

34 Bracher, Auflösung der Weimarer Republik, S. 431 ff.; vgl. auch Horn, a. a. O., S. $335 \mathrm{f}$.

35 Personalakte Lenz, BDC, Bericht vom 26.1.1934.

36 Personalakte Lenz, BDC, Belegexemplare der „Algrena“ vom 21.12., 24.12., 28. 12. 1932.

37 Das genaue Datum des Rücktritts ist nicht zu ermitteln. Im Tagebuch von Felix Wankel taucht die Nachricht unter dem 17. 12. 1932 auf. Personalakte Lenz, BDC. 
Festigung der „Notgemeinschaft" wurden von ihm Bevollmächtigte auf regionaler Ebene bestimmt, die etwa den Kreisleitern der NSDAP entsprachen.

Im ersten Artikel „Ich habs gewagt“, der am 21. Dezember 1932 in der „Algrena“ erschien, schrieb Lenz noch recht nebelhaft. Unter Hinweisen auf den Freiherrn vom Stein, Graf York von Wartenburg, Ulrich von Hutten und Franz von Sickingen verherrlichte er den Rebellen, der ,die Fahne weitertragen (werde)“ zum Siege der „Revolution des deutschen Nationalismus und Sozialismus"; den Namen Straßer nannte er nicht. In der nächsten Ausgabe vom 24. Dezember 1932 wurde er deutlicher. Unter dem Titel „Hitler oder Straßer“ ließ er einen linientreuen Bonzen mit einem ,hitlertreuen Oppositionellen“ diskutieren. Ebenso wie Stegmann in Franken und schon früher Stennes berief sich auch die „Notgemeinschaft" auf die Person Hitlers und behauptete, der wahre Träger der Hitler-Idee zu sein. Das eigentliche Übel, so wurde erklärt, sei die ,byzantinierende“ Umgebung Hitlers, die den Führer längst der wirklichen Lage des Volkes und der Partei entfremdet und ihn daher seiner Unabhängigkeit beraubt habe. Lenz ließ den Oppositionellen sagen: „Schau seine (Hitlers) Umgebung an - betrachte Dir seine Berater und Einflusser - die führen ihn in Massenversammlungen, und im Tosen des Beifalls, unter Bergen von Blumen und im Banne von Kinderaugen, fanatischen Frauen, einsatzbereiten Männern, sagt ihm sein Gefolge - so treu stünde diese Masse zur Partei. Keiner von diesen Kerlen wagt es, dem Führer reinen Wein einzuschenken - und die Wahrheit zu sagen.“ Straßer wurde dagegen als ,,revolutionärer Kämpfer mit glasklarem Urteil" bezeichnet, der alleine noch in der Lage sei, die Bewegung zu erneuern und zu erhalten: „Hitler muß es hören, wie es in Wirklichkeit ausschaut, und Straßer hat das einzig Mögliche getan. Wir tun es ihm nach. Noch hat Hitler die Liebe des Volkes. Damit aus Liebe nicht $\mathrm{Ha} ß$ wird, darum stehen wir in Opposition. Wir sind die Alten geblieben aus Treue zum Führer und im Glauben an Deutschland.“ Am 28. Dezember 1932 veröffentlichte Lenz seinen dritten Artikel unter dem Titel „Der Fall Straßer“. Auf die Umgebung Hitlers anspielend, vor allem auf Goebbels, schrieb er, $\mathrm{da} ß$ die Entwicklung der letzten Wochen den Sieg ,,der falschen Hitlerpaladine über den Soldaten und Mann Straßer" gebracht habe. „Heute dürfen solche Elemente schreiben, es sei gleichgültig, ob ein Gregor Straßer aus der Bewegung ging oder nicht. Gregor Straßer trug um Hitler schon Narben, als jene noch intellektuelle Fassadenklettereien vollführten “. ${ }^{38}$ Zum Schluß erklärte er: .. , das Herz der Nationalsozialisten schlägt für Straßer. Das Herz und die Kraft der Bewegung hoffen, daß Straßer zurückkehren wird. Die Bedingung, die er stellen wird, und die er stellen muß, ist die Wiederherstellung der alten Linie, die Úbernahme der Führung durch die alte Garde ...." Das war allerdings die letzte Ausgabe der „Alemannischen Grenzlandnachrichten“. Offensichtlich um Einfluß auf den Inhalt des Blattes zu bekommen, waren die Herausgeber vom Verleger unter finanziellen Druck gesetzt worden. Es ist zu vermuten, daß dahinter politische Einflüsse standen, wahrschein-

38 Gemeint ist der Artikel zum Ausscheiden Straßers in der Berliner Gauzeitung „Der Angriff" vom 10.12.1932, von dem sich Goebbels später distanzieren mußte. 
lich von Seiten der offiziellen NSDAP. Am 31. Dezember 1932 wurden diese Zusammenhänge den „Lesern und Abonnenten“ der Algrena durch ein Rundschreiben erklärt, nicht ohne die Ankündigung, daß bald ein Nachfolgeorgan erscheinen werde. ${ }^{39}$

Am 7. Januar trat „Die Notgemeinschaft, Nachrichten- und Kampfblatt der NSN“, ein wöchentliches Mitteilungsblatt, diese Nachfolge tatsächlich an. In der Begrüßung der Leser wurde auf die Bildung zweier neuer Ortsgruppen in Baden hingewiesen. Dann folgte ein Versuch, die „Notgemeinschaft" in das Parteispektrum einzuordnen. Im völkischen Lager, so hieß es, gebe es außerhalb der NSDAP noch drei Parteien. „1. Die Revolutionären Nationalsozialisten unter Führung von Dr. Otto Straßer, 2. die Deutschsozialistische Partei, und 3. die deutschvölkische Freiheitsbewegung unter Graefe, Wulle, Henning“. Während die Otto-Straßer-Bewegung als konsequent antiparlamentarische Bewegung kaum als Bündnispartner in Frage kam, war die deutschvölkische Partei Graefes als konservativ-monarchistische Gruppe für die „Notgemeinschaft" nicht tragbar. Die Deutschsozialistische Partei jedoch, die im wesentlichen aus ehemaligen Nationalsozialisten bestehe und die jetzt durch den Anschluß der früheren Deutsch-Sozialen unter Knüppel-Kunze verstärkt worden sei, so sagte die „Notgemeinschaft“, könne ein Partner sein: „Die Führer der Deutschsozialisten haben uns in einem Schreiben mitgeteilt, daß sie sich geschlossen mit ihren Anhängern hinter Gregor Straßer stellen würden, wenn dieser handeln würde. Die gleiche Erklärung haben auch zahlreiche Abgeordnete der NSDAP, angewidert von dem Byzantinismus und undeutschem Gehorsam in der Partei, abgegeben. Ebenso werden sich die Notgemeinschaften in Baden geschlossen für Gregor Straßer erklären. Welchen Weg die weitere Entwicklung nehmen wird, werden wir in den nächsten Wochen sehen. Wir erinnern uns an das offene Wort, das Gregor Straßer Hitler gegenüber vor seinem Abgang gebraucht hat, daß es gar keinem Zweifel unterliege, daß die NSDAP bei Neuwahlen, d.h. wenn Schleicher seine Drohung, den Reichstag aufzulösen, wahrmachen würde, wiederum 2 Millionen Stimmen verlieren würde."

In großen Aufrufen wurden die SA- und SS-Männer aufgefordert, darüber nachzudenken, für welche Parteibonzen sie arbeiteten und bluteten. ${ }^{40}$ Aus den offensichtlich vielen Úberläufern - exakte Zahlen fehlen allerdings - wurde Ende Januar 1933 in Anlehnung an das Beispiel Stegmanns, mit dem man auch in Kontakt getreten war, ein „Freikorps Oberrhein“ gegründet, ${ }^{41}$ außerdem eine „Frauenschaft" geschaffen $^{42}$ und schon bald der regelmäßige Sprechabend eingeführt. Außer zu Stegmann bestanden enge Beziehungen zu einer schnell wachsenden Berliner NS-Opposition unter Leitung von Wilhelm Klutz, ${ }^{43}$ in deren Organ „Der deutsche Weg“

39 Erklärung der „Algrena“ vom 31. 12. 1932 in der Personalakte Lenz, BDC.

40 „Die Notgemeinschaft“", 7.1.1933, S. 6, 14.1.1933, S. 6.

41 A. a. O., 4. 2. 1933, S. 1.

42 A. a. O., 28. 1. 1933, S. 1.

43 A. a. O., 14. 1. 1933, S. 2. 
unter anderem der Vertraute Straßers Reupke schrieb, ${ }^{44}$ und nach Sachsen, wo sich aus „Massenaustritten aus der NSDAP“ ebenfalls eine Opposition zu bilden begann. ${ }^{45}$

Über die Wahlen in Lippe am 15. Januar 1933 wurde in der „Notgemeinschaft“ unter der Überschrift „Der Pyrrhussieg von Lippe“ berichtet. ${ }^{46}$ Die Wahl habe vor allem eine taktische Bedeutung, und zwar vornehmlich im innerparteilichen Bereich, wo durch einen Erfolg die deprimierten Anhänger wieder aufgerichtet werden sollten. Indes wurde bezweifelt, daß die NSDAP wirklich einen Sieg errungen habe; im Verhältnis zum riesigen Wahlaufwand der NSDAP, die ihre „Kanonen Hitler, Goebbels, Lutzmann, Göring " selbst in die kleinsten Ortschaften und Versammlungen geschickt habe, sei der Erfolg recht bescheiden ausgefallen. Zwar habe die Partei in Lippe den Tiefstand an Stimmen vom 6. November 1932 (39056) um rund 6000 Stimmen übertroffen, aber das Ergebnis vom 31. Juli 1932 immer noch um 3000 Stimmen verfehlt - und noch nicht einmal das damalige Wahlergebnis habe ja zur Machtübernahme gereicht. Da aber die NSDAP im Reich nicht zu einem solchen Aufwand wie in Lippe fähig sei, könnten ,weder die Siegesfanfaren noch die neuen Kampfansagen gegen Schleicher darüber hinwegtäuschen, daß eine Reichstagsauflösung und Neuwahlen keiner Partei so unangenehm wären wie der NSDAP." In der Ausgabe vom 4. Februar 1933, also nach der Machtübernahme Hitlers, die mit Skepsis betrachtet wurde, machte dann „Die Notgemeinschaft" ihrer Enttäuschung über Straßers Zögern Luft: „Hätte Straßer seine Schicksalsstunde erkannt, hätte er gehandelt, hätte er den offenen Kampf gegen die NSDAP im Rahmen einer eigenen Partei und scharfer und klarer Heraushebung der positiven und taktischen Ziele aufgenommen, dann wäre ihm die Gefolgschaft aller Oppositionsgruppen sicher gewesen, und er hätte eine große Aufgabe und eine für Deutschland segensreiche Mission erfüllen können. Aber in der entscheidenden Stunde hat er versagt. Sein Zögern, seine Unentschlossenheit und seine freiwillige Unterwerfung haben die starke Wirkung, die von seiner Persönlichkeit ausging, genommen. Von einem Führer werden klare und entschlossene Entscheidungen, aber keine taktischen Bauchtänze verlangt.“ „Die Notgemeinschaft" erschien noch einige Male - die technische Aufmachung der beiden letzten Nummern war schon recht schlecht - und endete mit der Ausgabe vom 11. März 1933, in der über die Wahlen vom 5. März 1933 berichtet wurde. Gleichzeitig löste sich auch die „Nationalsozialistische Notgemeinschaft" als Organisation wieder auf.

Im Juli 1933 begann ein Parteigerichtsverfahren gegen das Haupt der Lahrer ,Notgemeinschaft", den ehemaligen Gauleiter Lenz. ${ }^{47}$ Die Untersuchung des Falles wurde der Gau-Uschla des benachbarten Gaues Rheinpfalz übertragen. Lenz versuchte zunächst, alles abzuleugnen bzw. als Bagatelle hinzustellen; er benannte auch

\footnotetext{
44 Personalakte Reupke, BDC.

45 „Die Notgemeinschaft", 14. 1. 1933, S. 1 f.

46 A. a. O., 21. 1. 1933, S. 1.

47 Personalakte Lenz, BDC.
} 
etliche Entlastungszeugen, vor allem höhere Parteifunktionäre aus seinem ehemaligen Gau Hessen-Darmstadt. In dem „Bericht über die Voruntersuchung zum Parteigerichtsverfahren gegen den Pg Karl Lenz M. d. R. in Heidelberg" vom 26. Januar 1934 stellte jedoch der Vorsitzende der Gau-Uschla fest, daß die geschilderten Vorgänge des Winters 32/33 erwiesen und die hessischen Entlastungszeugen offenbar selbst in die Affäre verwickelt gewesen seien. Merkwürdigerweise setzte er an den Schluß des Untersuchungsberichtes noch eine eigene Stellungnahme, zu der er, wie er selbst bemerkte, satzungsgemäß gar nicht befugt war. Darin bat er um eine milde Beurteilung des Falles und erklärte, daß es für Lenz zu hart sei, aus der Partei ausgeschlossen zu werden. Zuvor hatte der gleiche Bericht Lenz einer schwerwiegenden Parteirevolte überführt. Dies lenkt den Blick auf den Gau Rheinpfalz.

\section{c) Der Gau Rheinpfalz}

Gauleiter Bürckel galt als besonderer Straßer-Freund, und der Gau Rheinpfalz wurde als "Straßer-Gau“ bezeichnet; Straßer besuchte ihn denn auch häufig zu Versammlungen. ${ }^{48}$ In den Tagen nach Straßers Ausscheiden suchte Gauleiter Bürckel alle wichtigen Funktionäre und Abgeordneten seines Gaues auf, um sie auf sich einzuschwören. Am 11. Dezember 1932, einem Sonntag, fand in Neustadt der Generalappell des Gaues Rheinpfalz statt, ${ }^{49}$ bei dem auch der neue Stabsleiter der ROL, Ley, anwesend war. Bürckel jedoch forderte in einer großen Rede die revolutionäre Verwirklichung ,des deutschen Sozialismus“ durch die NSDAP, mehrfach betonend, daß alle alten Kämpfer „,deutsche Sozialisten“ seien, womit er typisches Vokabular des Straßerflügels aufgriff. Im nichtöffentlichen Teil der Tagung sagte er vor den Kreisleitern und in Anwesenheit von Ley, ,,er werde jeden in seinem Gau in Fetzen reißen, der es wage, etwas gegen Gregor zu sagen oder etwas zu unternehmen“. ${ }^{50}$ Zusätzlich ließ er sich von allen Kreisleitern Treue geloben und versichern, „daß er immer den richtigen Weg in seinem Gau gegangen sei, den er auch in Zukunft gehen werde". Offenbar hatte Bürckel die Funktionäre und, soweit erkennbar, auch die Abgeordneten seines Gaues tatsächlich hinter sich. Nur der landwirtschaftliche Gaufachberater und Reichstagsabgeordnete Schickert opponierte, der bisher mit Bürckel eng befreundet war. Jetzt entzündete sich an dem unterschiedlichen Verhältnis zu Straßer eine tiefe Feindschaft, die im Laufe des Frühjahrs 1934 soweit führte, daß Bürckel versuchte, Schickert aus der Partei auszuschließen. Schickert schloß sich stärker an den Reichsbauernführer Darré an und strengte im Herbst 1934 seinerseits ein Parteigerichtsverfahren gegen den Gauleiter wegen dessen Haltung in der Straßerfrage an. Aus seiner Tätigkeit als Reichstagsabgeordneter, so gab Schickert dabei an, wisse er, daß die Gauleiter Lohse, Koch, Karpenstein und Bürckel ebenso wie der Pfälzer Reichstagsabgeordnete und Freund Bürckels Selzner

48 Personalakte Bürckel, BDC: Aussage Schickert MdR, Aussage Leyser, stellvertr. Gauleiter und Vorsitzender des Gaugerichts.

49 VB, 16. 12. 1932.

so Personalakte Bürckel, BDC: Aussage MdR Schickert. 
in engem persönlichen Kontakt zu Straßer gestanden hätten, der auch noch nach dessen Ausscheiden als Reichsorganisationsleiter fortgesetzt worden sei; Selzner habe kurz vor den Lippe-Wahlen geäußert, ,man wisse nicht, was besser sei, ob die Wahlen gut oder schlecht ausgingen. Falls sie gut ausfielen, dann bekommen die Herren in Berlin den Größenwahnsinn".

Die pro-Straßer-Stimmung im Gau Rheinpfalz, besonders beim Gauleiter selbst, hielt offensichtlich bis 1934 unvermindert an. Am 30. Juni 1934 fand dort in Anwesenheit von Darré eine große Bauernversammlung statt. Nach einem persönlichen Affront gegen Schickert griff Bürckel am Schluß seiner Eröffnungsrede auch noch den Reichsbauernführer so scharf an, daß Darré nach der Kundgebung äußerst verletzt abfuhr. Am nächsten Tag, also am 1. Juli 1934, rief Darré bei der Gauleitung an und ließ Bürckel bestellen, ,daß das Schlußwort des Gauleiters Bürckel auf dem Donnersberg ihm so gefallen hätte, daß er nicht umhin könne, Gauleiter Bürckel mitzuteilen, daß soeben Gregor Straßer erschossen worden sei “ ${ }^{51}$ Bürckel, nun offensichtlich verängstigt, rief daraufhin Darré mehrfach an, um sich für seine Rede zu entschuldigen; auch suchte er sich sofort Rückhalt im eigenen Gau zu verschaffen, indem er Zeugen sammelte, die aussagen sollten, daß er seit der Gauleitertagung vom 17. Januar 1933, also unmittelbar nach den Lippe-Wahlen, keinen Kontakt mehr mit Straßer gehabt habe.

\section{Straßer nach seinem Ausscheiden aus der ROL}

Gregor Straßer war am 8. Dezember 1932, als er alle Parteiämter niederlegte, Mitglied der NSDAP geblieben. Entgegen der Ankündigung in seinem Rücktrittsbrief, er wolle auch sein Reichstagsmandat zur Verfügung stellen, behielt er es jedoch bis zum Ende der Wahlperiode am 5. März 1933. Dies hatte vor allem zwei Gründe. Einmal hätte er dann seine Immunität verloren, was ihm am 8. Dezember noch sehr unangenehm gewesen wäre. Aus den Zeiten des Kampfverlages verfolgten ihn nämlich noch eine Reihe von Prozessen wegen Beleidigung politischer Gegner und Vergehen gegen das Republikschutzgesetz, ${ }^{52}$ die zum Teil bereits zu rechtskräftigen Verurteilungen geführt hatten, deren Vollstreckung nur durch sein Reichstagsmandat verhindert wurde. Die Prozesse dürften auch ein wesentlicher Grund für seine Mitgliedschaft im Reichstagsausschuß zur Wahrung der Rechte der Volksvertreter gewesen sein. ${ }^{53}$ Es gehörte zu den wichtigsten Rechten dieses Ausschusses, daß er als einziger Reichstagsausschuß auch nach einer Auflösung des Reichstages weiterbestand und bis zur Neuwahl als eine Art Notparlament arbeitete. Den Ausschußmitgliedern sicherte das in einer Zeit häufiger Parlamentsauflösungen eine

51 Ebenda.

52 Siehe oben S. 43.

53 Von Frick am 4. 1. 1933 angefertigte Liste der NS-Obleute in den einzelnen Reichstagsausschüssen, BA, Slg. Schumacher/318. 
gewisse politische Einflußmöglichkeit, für Straßer bedeutete es aber auch den Schutz vor dem Zugriff der Gerichte, nämlich permanente Immunität. Erst mit dem Amnestiegesetz, das der Reichstag am 21. Dezember 1932 erließ, erledigten sich für ihn diese Prozesse und ihre Folgen. Der zweite Grund, sein Reichstagsmandat zu behalten, bestand für Straßer vermutlich darin, daß er sich die Möglichkeit zur Rückkehr in die Politik offenhalten wollte.

Als er zu Weihnachten 1932 von seiner Erholungsreise nach Bozen zurückkehrte, hatte er sich mit dem Gedanken eines Wiedereintritts in die Politik bereits angefreundet und nahm daher sofort Kontakt zu den verbliebenen Freunden auf. Zwischen Weihnachten und Neujahr traf er mit Brüning in Freudenstadt zusammen und sprach sich mit ihm aus. ${ }^{54} \mathrm{Zu}$ diesem Zeitpunkt war er schon in der Lage, Brüning auf ein Treffen Papen/Hitler Anfang Januar im Hause des Barons Schroeder aufmerksam zu machen. Den Vorschlag Brünings jedoch, er solle sich sobald wie möglich mit Hitler aussöhnen und wieder in seine Stellung in der Partei zurückkehren, um Schlimmeres zu verhüten, lehnte Straßer als unmöglich ab. Im weiteren Verlauf des Gesprächs versicherte ihn Brüning seiner grundsätzlichen Unterstützung, warnte ihn zugleich aber vor kritiklosem Glauben an Versprechungen Schleichers. Straßer hatte auch wieder Kontakte nach Berlin herstellen können, und zwar über seine Vertrauten Reupke ${ }^{55}$ und Elbrechter. ${ }^{56}$ Von Elbrechter war er auch über das geplante Treffen Papen/Hitler am 4. Januar 1933 informiert worden. Elbrechter hatte überdies Schleicher vorgewarnt, jedoch mochte der Reichskanzler an diese Information nicht so recht glauben. So schickte Elbrechter privat einen Fotografen nach Köln und ließ Hitler und Papen dort fotografieren. ${ }^{57}$ Die Bilder erschienen am nächsten Tag, als innenpolitische Sensation aufgemacht, in der „Täglichen Rundschau“. Ebenfalls am 4. Januar 1933 ließ Schleicher Gregor Straßer beim Reichspräsidenten Hindenburg als zukünftigen Vizekanzler vorstellen. Hindenburg, sichtlich von Straßer angetan, stimmte der geplanten Ernennung zu. ${ }^{58}$ Schleicher jedoch, geschockt durch das Kölner Treffen, wartete in leicht resignierender Haltung zunächst ab, ohne die Ernennung Straßers sofort zu vollziehen, die auf die Partei Anfang Januar nicht ohne Signalwirkung hätte bleiben können. Gregor Straßer, seit dem Tage seines Rücktritts in jene merkwürdige resignative Inaktivität gefallen, war offensichtlich ebenfalls froh, sich wieder nach München zurückziehen und weiter abwarten zu können, zumal in ihm ein gewisses Mißtrauen gegen Schleicher langsam stärker wurde. ${ }^{59}$ Erst in der Ministerbesprechung vom 16. Januar 1933 ließ Schleicher über die Einbeziehung Straßers ins Kabinett diskutieren, wobei er klarmachte, daß Straßer Mitglied der NSDAP bleiben wolle. ${ }^{60}$ Am Tag zuvor, also am 15. Januar

54 Brüning, a. a. O., S. $639 \mathrm{f}$.

55 Personalakte Reupke, BDC.

56 Elbrechter-Nachlaß.

57 Elbrechter-Nachlaß; Auskunft Elbrechter jr. v. 20. 10. 1971; Treviranus, a. a. O., S. 355.

58 Otto Meissner, Staatssekretär unter Ebert-Hindenburg-Hitler, Hamburg 1950, S. $251 \mathrm{f}$.

59 Brief Heinrichsbauer an Reupke vom 13. 1. 1933, Personalakte Reupke, BDC.

60 BA, Reichskanzlei, Bd. 1459. 
1933, war jedoch, wie bereits erwähnt, der Landtag des kleinen Landes Lippe neugewählt worden. Das Wahlergebnis stellte, ohne allerdings das Ergebnis vom 31. Juli 1932 erreichen zu können, einen Erfolg der NSDAP dar, der es der Parteiführung erlaubte, sowohl gegenüber der Öffentlichkeit als auch intern wieder Siegesparolen auszugeben; Hitlers Stellung festigte sich. Am gleichen Tag, an dem der Ministerrat die Ernennung Straßers erörterte, konnte Hitler auf einer internen Gauleitertagung in Weimar, mit dem Wahlsieg im Rücken, den Gauleitern Mut einflößen und sie dadurch wieder fester an sich binden ${ }^{61}$ So glaubte auch Schleicher zu diesem Zeitpunkt nicht mehr, daß Straßer viel Anhang mitbringen würde. In Schleichers Augen hatte Straßer aber, neben seiner persönlichen Leistungsfähigkeit, noch immer zwei nicht zu übersehende Vorteile anzubieten. Das Ansehen, das Straßer in der SA, vor allem auch in Preußen, besaß, hätte es Hitler erschwert, wenn nicht sogar unmöglich gemacht, die SA geschlossen zu einem Staatsstreich gegen eine Regierung Schleicher-Straßer zu führen, ${ }^{62}$ falls die Regierung etwa durch Reichstagsauflösung und langes Hinausschieben der Neuwahlen in die formale Illegalität gegangen wäre. Eine solche Befürchtung hatte dem sogenannten „Planspiel Ott“" zugrunde gelegen. ${ }^{63} \mathrm{Der}$ große Ruf, den Straßer in der NSDAP, aber auch in anderen bürgerlichen Kreisen genoß, bot aber außerdem die Möglichkeit, bei Neuwahlen eine eigene Liste „StraBer" aufzustellen. ${ }^{64}$

Straßer selbst beschäftigte sich jedoch bereits mit dem Gedanken an einen zivilen Beruf. Etwa seit Mitte Januar suchte Reupke für ihn im Berliner Raum eine passende Apotheke oder eine entsprechende Position, jedoch zunächst erfolglos. ${ }^{65}$ Seit Ende Januar 1933 bemühte sich Straßer auch um eine Aussprache mit Hitler, von der er sich zwar keine politischen Gewinne versprach, die aber seine persönliche Lage klären und ihm den Eintritt in einen Beruf erleichtern konnte. ${ }^{66}$ Vor allem Frick und Funk waren ihm bei diesem Versuch behilflich; auch Hess wurde von Straßer eingeschaltet, doch offenbar vergeblich. Hitler war für ihn nicht zu sprechen. Seit April 1933 bemühte sich noch Pietsch ${ }^{67}$ um Straßer. In Gesprächen mit Hitler brachte er das Problem Straßer zur Sprache, da ohne das Wohlwollen des Führers Straßer nur schwer in einer entsprechenden Stellung in der Wirtschaft unterzubringen war. ${ }^{68}$ Offenbar im Frühsommer 1933 erhielt Pietsch von Hitler tatsächlich das Einverständnis mit einer Direktionsstelle für Straßer bei der Firma Schering-Kahlbaum in Berlin. ${ }^{69}$ Straßer mußte sowohl dem Vorstand der Firma als auch der

61 VB, 18. 1. 1933.

62 „Abegg-Bericht“", S. 13, IfZ, F 86.

63 „Planspiel Ott", IfZ, F 41, Bd. 4, Dokument 38.

${ }^{64}$ Elbrechter-Nachlaß; Personalakte Reupke, BDC.

65 Brief Reupke an Straßer vom 28. 1. 1933, Personalakte Reupke, BDC.

66 Ebenda.

67 Personalakte Pietsch, BDC.

68 Brief Pietsch an Reupke vom 15. 4. 1934, Personalakte Reupke, BDC.

69 Laut Auskunft der Schering AG ist eine Personalakte Gregor Straßer nicht mehr vorhanden (Brief vom 23.4. 1971). 
Parteileitung gegenüber schriftlich versichern, sich jeglicher politischer Tätigkeit und, soweit er das geschäftlich irgendwie konnte, auch nahezu aller privaten und gesellschaftlichen Kontakte zu enthalten. Am 1. Februar 1934 bekam er von Reichsschatzmeister Schwarz das goldene Parteiabzeichen der NSDAP verliehen.$^{70}$ Mit Genehmigung Hitlers übernahm er die unpolitische, aber ehrenvolle Position eines „1. Vorsitzenden der Reichsfachschaft der Pharmazeutischen Industrie“. So schien sich alles um ihn beruhigt zu haben. In der Presse wurde er nicht mehr erwähnt, seine berufliche Position sicherte ihm und seiner Familie ein gutes Auskommen; er konnte auch noch seine Mutter und seine Schwiegereltern unterstützen. Er arbeitete sich in seinem neuen Beruf allmählich ein und begann seine bürgerliche Tätigkeit langsam interessanter zu finden.

Doch im Frühjahr 1934 bekam er offensichtlich Warnungen zugespielt, daß seine Rolle wieder im Gespräch sei. Anlaß dazu war die Verhaftung von Dr. Reupke, seines Vertrauten vom Winter 1932/33, der zu den wenigen Personen gehörte, mit denen er auch jetzt noch losen privaten Kontakt hatte; gleichzeitig wurde gegen Reupke ein Parteigerichtsverfahren eingeleitet. Am 18. Juni 1934 schrieb Straßer daher einen fünfseitigen Brief an Hess, in dem er zunächst die Bedeutung Reupkes herunterspielte, dann aber auf seine eigenen Probleme einging. ${ }^{71}$ In ängstlichem und fast weinerlichem Ton beteuerte er, sich nie wieder politisch betätigt zu haben und dies auch in Zukunft nicht tun zu wollen. Auch stritt er jede Verbindung zu seinem im Exil lebenden Bruder Otto ab und bat Hess, ihm doch Glauben zu schenken: „Heute bitte ich Sie um Auskunft, was ich nach Ansicht der Partei tun soll, um die in meinen verschiedenen Erklärungen abgegebene und von mir restlos durchgeführte politische Abstinenz auch nach außen hin deutlich sichtbar zu machen. Ich bin in dieser Hinsicht bereit, jedes Verlangen und jede Forderung, die die Partei an mich stellt, zu erfüllen, denn auch ich will nichts mehr, als ohne Angriff und Mißdeutung meine neue Lebensarbeit aufbauen und niemals werde ich irgend etwas tun, was der Partei schaden könnte. Wenn man die Tatsache, daß ich erster Vorsitzender der Reichsfachschaft der Pharmazeutischen Industrie bin, als ein nicht erwünschtes Hervortreten in der Öffentlichkeit bezeichnet, so bitte ich um eine diesbezügliche Nachricht, um dann meinen Rücktritt zu vollziehen. Wenn irgendeine andere Maßnahme für notwendig gehalten wird, wenn aus Gründen, die ich letzten Endes sogar verstehen kann, eine genaueste UUberwachung meiner Person und meiner Tätigkeit für notwendig gehalten wird, so bin ich damit deswegen ohne weiteres einverstanden, weil ich weiß, daß eine sachliche und objektive Úberwachung auch im letzten Jahr der beste Beweis für die Richtigkeit meiner Darlegungen und die genaueste Einhaltung meiner Erklärung sein würde.

Kurzum, ich bitte auf Grund einer zehnjährigen, opferfreudigen und einsatzbereiten Tätigkeit beim Aufbau der Partei um ihren Schutz und um die Bekanntgabe dessen, was ich tun soll, um im Sinne meiner Erklärung aus jeder Debatte zu verschwinden

70 Besitzurkunde, Personalakte Gregor Straßer, BDC.

71 Brief G. Straßer an Hess vom 18.6. 1934, Personalakte Pietsch, BDC. 
und vor allen Dingen das mich unsäglich verletzende und infamierende Gefühl einer feindlichen Einstellung zur Partei zu beseitigen.

Ich bitte um Entschuldigung, daß ich Ihre Zeit so lange in Anspruch genommen habe, ich bitte aber andererseits auch um Verständnis für meine kaum mehr zu ertragende Lage."

Nach diesem Brief fühlte sich Straßer offenbar wieder einigermaßen sicher, trotz der Warnungen, die er von den verschiedensten Seiten, unter anderem von Brüning, ${ }^{72}$ erhielt. Wenige Tage später bat er jedenfalls die Familie Höfler, seine Schwester und seinen Schwager, Ende Juni von Bayern nach Berlin zu Besuch zu kommen und dort einige Tage zu verweilen. ${ }^{73}$ Die Einladung konnte zwar nicht angenommen werden, doch hofften alle, sich anläßlich des Urlaubs, den Gregor Straßer für August plante, wiederzusehen.

Am 30. Juni 1934, einem Samstag, saß die Familie Straßer am Mittagstisch in der Berliner Privatwohnung, Müllerstraße 170/71. ${ }^{74}$ Unerwartet erschienen fünf Beamte der Gestapo in Zivil und teilten ihm mit, daß er sofort mit ihnen zu kommen habe, da sie eine Haussuchung in seinem Büro bei Schering-Kahlbaum durchführen müßten. Nachdem Straßer seine Familie beruhigt hatte, fuhr er mit den Beamten. Anstatt ihn in sein Büro zu bringen, übergaben sie ihn jedoch an ein SS-Kommando, das mit ihm ins Gebäude der Geheimen Staatspolizei in der Prinz-Albrecht-Straße fuhr. ${ }^{75}$ Bereits gefesselt, wurde er von drei mit Maschinenpistolen bewaffneten Schergen an etlichen anderen Verhafteten vorbei in eine Einzelzelle des Kellergeschosses gebracht und sofort mit mehreren Pistolenschüssen durch einen SS-Hauptsturmführer ermordet, der den Keller dann mit den Worten verließ: „Das Schwein wäre erledigt." Die Leiche wurde offenbar an Ort und Stelle in Einzelteile zerstükkelt und in mehreren blutigen Säcken weggebracht.

Eine Woche später, am 7. Juli 1934, wurde Frau Straßer eine Urne in die Wohnung gebracht mit der Aufschrift: „Gregor Straßer, geboren 30.5.92 zu Geisenfeld, ist am 30.6. 34 um 17.20 gestorben. Geheime Staatspolizei Berlin." ${ }^{\text {"76 }}$ Da der Familie gleichzeitig dringendst nahegelegt wurde, kein öffentliches Aufsehen zu erregen, unterblieb die Beisetzung der Urne. Der Familie zufolge befindet sie sich noch heute in der Wohnung der Witwe Else Straßer.

Offiziell war als Todesursache, wie bei anderen Opfern des 30. Juni 1934, „Selbstmord" angegeben worden. ${ }^{77}$ Frau Straßer, die ohne materielle Grundlage dastand und zusätzlich für ihre beiden Söhne sorgen mußte, geriet dadurch in zusätzliche Schwierigkeiten. Die abgeschlossenen Lebens- und Unfallversicherungen enthielten nämlich die Klausel, daß bei Selbstmord keine Zahlungsverpflichtung der Versiche-

72 Eidesstattliche Erklärung Brünings vom 10.1. 1953, Elbrechter-Nachlaß, siehe Anhang.

73 Auskunft Frau Höfler, geb. Straßer v. 10.4. 1971. Sie besitzt auch noch die entsprechende schriftliche Einladung Straßers.

74 Otto Straßer, Die deutsche Bartholomäusnacht, Prag/Zürich/Brüssel 1938, S. 44ff.

75 Fritz Günther v. Tschirschky, Erinnerungen eines Hochverräters, Stuttgart 1972, S. 195 f.

76 O. Straßer, Bartholomäusnacht, S. 46.

77 „Amtliche Totenliste vom 30. Juni 1934“, IfZ, MA 131, Bl. 103458-64. 
rungen bestehe. ${ }^{78}$ Frau Straßer wandte sich, als sich die Versicherungen in der Tat weigerten, die Beträge auszuzahlen, an den Reichsinnenminister Frick, dem es nach etlichen Schwierigkeiten, vorsichtig an der Gestapo vorbeiagierend, gelang, die führenden Herren der beiden Versicherungsgesellschaften zur Freigabe der Gelder zu bewegen, ohne daß er von der Fiktion des „Selbstmordes“ offen abging. Da das schriftlich zu schwierig war, ließ er die entscheidenden Informationen durch geeignete Mitarbeiter des Ministeriums mündlich übermitteln.

Seit 1. Mai 1936 wurde Frau Straßer zusätzlich vom Reichsführer SS Heinrich Himmler unterstützt, ${ }^{79}$ der ihr eine Rente von monatlich $500 \mathrm{RM}$ auszahlen ließ, wovon $300 \mathrm{RM}$ für sie selbst bestimmt waren und für jeden der beiden Söhne weitere $100 \mathrm{RM}$. Auch danach hielt Himmler offensichtlich seine Hand über sie. $\mathrm{Ob}$ es darum ging, Krankenhauskosten von über 1500,- RM zu übernehmen, ihr Bescheinigungen für die Befreiung von einer Haussteuer zu senden oder ob er sich dafür verwandte, daß die beiden Söhne Günther und Helmut in die Offizierslaufbahn des Heeres übernommen wurden, stets reagierte er auf entsprechende Bitten von Frau Straßer oder ihres Bruders Vollmuth ${ }^{80}$ positiv. Auch als nach dem Tod ihrer Söhne, die am 30. Juli 1941 bzw. am 27. Mai 1942 im Osten gefallen waren, zunächst 1600 RM Rente zuviel gezahlt worden waren, entschied er sofort, daß das Geld nicht zurückzufordern sei, die Rente aber jetzt auf $300 \mathrm{RM}$ festgesetzt werden sollte. In einem Brief vom 18. Oktober 1942 an die "Sehr verehrte Frau Straßer“ entschuldigte er sich, daß er nicht früher habe schreiben können und erst jetzt ,zu dem Heldentode Ihres Jungen ... herzliche Anteilnahme und herzliches Gedenken“ ausspreche. Sobald sein Osteinsatz vorbei sei und er wieder nach Berlin zurückkomme, wolle er ihr einen Terminvorschlag für eine Rücksprache machen. ${ }^{81}$

\footnotetext{
78 Einzelheiten hierzu bei Lothar Gruchmann (Hrsg). Erlebnisbericht Werner Pünders über die Ermordung Klauseners am 30.6. 1934 und ihre Folgen; in: VfZ 19 (1971), S. $407 \mathrm{ff}$.

79 Personalakte Else Straßer, BDC.

${ }^{80}$ Vollmuth war 1. Adjutant des Reichsorganisationsleiters Straßer.

81 Personalakte Else Straßer, BDC.
} 